\title{
Severe and early stenosis of porcine heterograft mitral valve
}

\author{
J. C. FORFAR, L. COTTER ${ }^{1}$, AND G. N. MORRITT \\ From the Departments of Cardiology and Cardiothoracic Surgery, The Royal Infirmary of Edinburgh, \\ Edinburgh, Scotland
}

SUMMARY A patient who had aortic and mitral valves replaced by Carpentier porcine heterografts for bacterial endocarditis developed severe heart failure 18 days after operation. A second emergency operation revealed that the mitral prosthesis had become severely stenosed and calcified. A loud Graham Steell murmur had developed during the 12 hours before reoperation but no distinct murmurs of mitral stenosis had been detected.

Mounted porcine aortic valve heterografts are being used in increasing numbers. Occasional reports have recorded cases of failure from valvular regurgitation, usually caused by a leaflet or leaflets becoming torn, sometimes in association with infective endocarditis. We report the development of severe mitral stenosis in a stent-muunted porcine heterograft necessitating emergency replacement 19 days after initial implantation.

\section{Case report}

A 26-year-old man with no previous history of serious illness or recent dental treatment was admitted to hospital with a 3-week history of fever, malaise, arthralgia, and weight loss. On examination, he looked ill with pallor ( $\mathrm{Hb} 12 \cdot 1 \mathrm{~g} / 100 \mathrm{ml}$ ), pyrexia $\left(38.5^{\circ} \mathrm{C}\right)$, and early finger clubbing. His pulse was $120 /$ minute, regular, of large volume and collapsing character. Systolic blood pressure was $160 \mathrm{mmHg}$ with sounds changing at $45 \mathrm{mmHg}$ but audible down to zero. The heart sounds were normal; grade $3 / 6$ midsystolic and early diastolic murmurs were maximal at the left sternal edge and a grade $3 / 6$ pansystolic murmur was maximal at the apex. Both liver and spleen were enlarged to $6 \mathrm{~cm}$ below the costal margin; no basal crepitations were heard and there was no peripheral oedema or elevation of jugular venous pressure. Urine microscopy revealed many

'Present address: Department of Cardiology, Brompton Hospital, Fulham Road, London. red blood cells. Electrocardiography showed sinus tachycardia and distinct left ventricular hypertrophy. An $x$-ray film of the chest showed no abnormality of heart size or shape and no evidence of pulmonary congestion (CTR 135/280).

A clinical diagnosis of infective endocarditis was supported by growth of Streptococcus viridans in all of nine consecutive blood cultures. The valvular lesions were judged to be regurgitation of both mitral and aortic orifices, the severity of the latter being of recent origin and the result of endocarditis. Arrangements were therefore made for elective surgical replacement of the aortic valve and the patient was prepared by bed rest and initiation of treatment of the infection with benzyl penicillin 4 megaunits 6-hourly intravenously and streptomycin $500 \mathrm{mg}$ 12-hourly intramuscularly. With this combination the patient's serum inhibited growth of his own organism at dilutions up to $1: 1000$. His general condition improved, his temperature settled, and operation was performed on the seventh day in hospital.

At operation the aortic valve was found to be tricuspid with all 3 cusps thickened and retracted. There were vegetations immediately below the noncoronary cusp and early abscess formation in the aortic root. The vegetations extended onto the aortic cusp of the mitral valve. Removal of the infection necessitated replacement of both aortic and mitral valves. Carpentier-Edwards stent-mounted porcine heterografts were used, $23 \mathrm{~mm}$ in the aortic position and $33 \mathrm{~mm}$ in the mitral position. 
Immediately after operation atrial fibrillation developed and crepitations were noted over the lung bases. Digoxin and diuretic therapy was started and parenteral penicillin and streptomycin continued. The heart sounds were normal and there was no more than a soft systolic murmur at the left sternal edge. Progress was satisfactory until the tenth postoperative day when pyrexia recurred. Eleven blood cultures were negative and though the pyrexia continued, no cause was found. On the thirteenth postoperative day the cardiac rhythm began varying between atrial fibrillation, junctional rhythm, and sinus rhythm with first degree heart block. At this time therapy was digoxin $0.25 \mathrm{mg}$ daily (with blood levels within the therapeutic range), frusemide 80 mg daily with potassium supplements, parenteral antibiotic as before (with serum inhibiting the patient's organism to a dilution of $1: 256$ ), and warfarin therapy with prothrombin ratios within the range 1.8 to $2.5: 1$ on the majority of occasions. On the sixteenth postoperative day cough with purulent sputum was evident, with no growth on culture, but in view of continued pyrexia, parenteral cloxacillin $1 \mathrm{~g}$ intravenously 6-hourly and gentamicin $80 \mathrm{mg}$ intravenously 8 hourly were added to treatment. Over the next 2 days there were increasing signs of left ventricular failure, decreasingly responsive to diuretic therapy. On the 18th postoperative day the patient developed cardiogenic shock, with drowsiness, tachypnoea, profuse sweating, cold extremities, oliguria, hypotension (BP $80 / 50 \mathrm{mmHg}$ ), and sinus tachycardia. The pulse was not collapsing in character but for the first time a grade 3/6 long early diastolic murmur became audible at the left sternal edge. The soft systolic murmur was unchanged but no other murmurs were heard. The severe left ventricular failure was attributed to aortic regurgitation caused by continued infection in the aortic root as evidenced by pyrexia and first degree heart block. The small pulse pressure and absence of a collapsing pulse were attributed to a low output state. Emergency surgery to replace the aortic prosthesis was decided upon and operation was performed in the early hours of the 19th day after the first operation.

At operation the pulmonary artery was noted to be tense but when the aorta was opened the aortic prosthesis appeared normal and no periprosthetic leak could be found. Even with strong suction applied to the left ventricular vent with saline in the aortic root there was no leakage of the saline through the valve. The left atrium was then opened and the mitral heterograft examined. The mitral prosthesis looked yellow in colour and when pressure was applied to the cusps it was found that there was strong resistance to opening. Though the nature of the change in the valve cusps was not apparent macroscopically, the valve was severely stenotic and was, therefore, excised. No thrombi or vegetations were present but the cusps were rigid and considerable force was required to open them. The mitral valve was replaced with a $31 \mathrm{~mm}$ Björk-Shiley tilting disc prosthesis. The aortic heterograft appeared satisfactory but in case the factor responsible for the mitral stenosis might later affect the aortic cusps it was decided that it too should be replaced by a BjörkShiley prosthesis $(23 \mathrm{~mm})$.

The patient's progress after the second operation was uneventful. Streptomycin, cloxacillin, and gentamicin therapy were discontinued one week after operation, but a 6 -week course of penicillin was completed. He was discharged home $3 \frac{1}{2}$ weeks after the second operation, maintained on oral anticoagulants but no other medical treatment. Six months later he was symptom free, in normal rhythm, blood pressue $130 / 75 \mathrm{mmHg}$ and had no murmurs or cardiac enlargement (CTR 130/280). The sounds of his prosthetic valves were satisfactory and his electrocardiogram was normal.

Examination of the prosthetic aortic valve showed it to be normal apart from some fibrin thickening of the leaflets at the centre of the valve. The mitral valve leaflets, however, were extremely hard and stiff. Histology showed a layer of calcium extending from the base of each leaflet through its entire length to the edge of the valve. Calcification was thicker at the tips of each leaflet (Fig. 1a), with bands extending out towards the base (Fig. 1b).

\section{Discussion}

Several long-term reports on the follow-up of mounted porcine heterografts in the mitral position have now been published. They all report some instances of valve failure which has usually been the result of regurgitation caused by perforation of cusps, detachment of cusps from their stents, or tears in the free margin of the cusps (Carpentier et al., 1974; Horowitz et al., 1974; Cohn et al., 1975; McIntosh et al., 1975; Stinson et al., 1977). Many of these instances of prosthetic valve failure have occurred in association with infective endocarditis. Several authors have also drawn attention to the presence of gradients across apparently normally functioning mitral porcine heterografts (Horowitz et al., 1974; Johnson et al., 1975). Overgrowth of fibrous tissue interfering with valve function has also been described (Horowitz et al., 1974; Stinson et al., 1974). Levitsky, in discussion following the report of Stinson et al. (1977) on 461 patients in whom porcine heterografts were implanted, described a 9-year-old child whose mitral porcine 

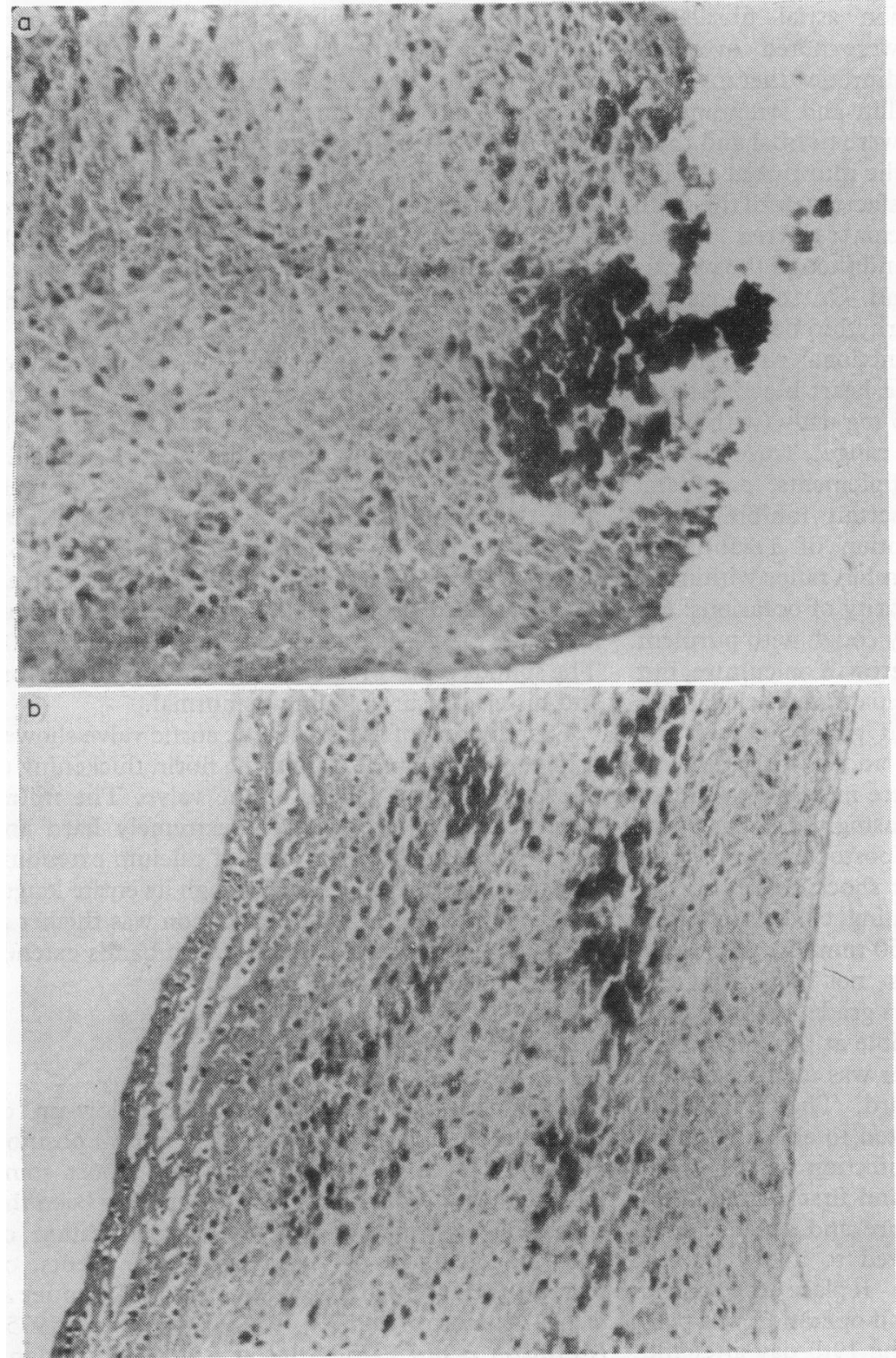

Fig. 1 Sections from the heterograft mitral valve showing heavy calcification (dark areas) at the tip of each leaflet (a) with bands of calcification extending towards the base (b). heterograft was removed 19 months after operation-all the cusps were stiff with areas of calcification on both atrial and ventricular surfaces.

There are few descriptions of histological examination of glutaraldehyde stabilised porcine heterografts removed after implantation. Yarbrough et al. (1973) examined 7 porcine heterograft valves removed from 6 patients and 1 calf between 2 and 26 weeks after implantation. All these valves were considered to be macroscopically normal but microscopically small foci of necrosis were present in 3 of them. There was no evidence of calcium deposition in any of the valves. Horowitz et al. (1974) examined 4 valves recovered between 4 and 29 months after operation and found preservation of cellular architecture and structural integrity in all valves, with little histological change as compared with unimplanted specimens. Zuhdi et al. (1974) have confirmed the paucity of histological change in valves prepared by the stabilised glutaraldehyde process and recovered up to 6 months after insertion. Severe and early stenosis of a heterograft in the 
mitral position has not been reported previously. The extent of the calcification within 19 days of insertion suggests that the heterograft valve itself may have had some defect which predisposed to its rapid deterioration.

It is clinically instructive that repeated auscultation had not suggested mitral valve dysfunction. The early diastolic murmur which first appeared 12 hours before the second operation increased to a grade $3 / 6$ intensity and was wrongly interpreted as recurrence of aortic regurgitation and an indication for urgent further surgery. The observations at operation excluded aortic regurgitation and provided grounds for concluding that the murmur had been the result of pulmonary regurgitation caused by pulmonary hypertension secondary to the severity of the mitral stenosis as described by Graham Steell (1888).

We are grateful to Dr R. M. Marquis and Mr P. R. Walbaum for advice and criticism in the preparation of this report.

\section{References}

Carpentier, A., Deloche, A., Relland, J., Fabriani, J. N., Forman, J., Camilleri, J. P., Soyer, R., and Dubost, C. (1974). Six year follow-up of glutaraldehyde-preserved heterografts. Fournal of Thoracic and Cardiovascular Surgery, 68, 771-781.

Cohn, L. H., Lambert, J. J., Castenada, A. R., and Collins, J. J., Jr. (1975). Cardiac valve replacement with the stabilised glutaraldehyde porcine aortic valve; indications, operative results and follow-up. Chest, 68, 162-165.

Horowitz, M. S., Goodman, D. J., Fogarty, T. J., and Harrison, D. C. (1974). Mitral valve replacement with the glutaraldehyde-preserved porcine heterograft. Fournal of Thoracic and Cardiovascular Surgery, 67, 885-895.

Johnson, A. D., Daily, P. O., Peterson, K. L., LeWinter, M., DiDonna, G. J., Blair, G., and Niwayama, G. (1975). Functional evaluation of the porcine heterograft in the mitral position. Circulation, 51 and 52, Suppl. 1, 40-48.

McIntosh, C. L., Michaelis, L. L., Morrow, A. G., Itscoitz, S. B., Redwood, D. R., and Epstein, S. E. (1975). Atrioventricular valve replacement with the Hancock porcine xenograft: a five year clinical experience. Surgery, 78, 768775.

Steell, G. (1888). The murmur of high pressure in the pulmonary artery. Medical Chronicle, 9, 182-188.

Stinson, E. B., Griepp, R. B., Oyer, P. E., and Shumway, N. E. (1977). Long term experience with porcine aortic valve xenografts. Fournal of Thoracic and Cardiovascular Surgery, 73, 54-63.

Stinson, E. B., Griepp, R. B., and Shumway, N. E. (1974). Clinical experience with a porcine aortic valve xenograft for mitral valve replacement. Annals of Thoracic Surgery, 18, 391-401.

Yarbrough, J. W., Roberts, W. C., and Reis, R. L. (1973). Structural alterations in tissue cardiac valves implanted in patients and in calves. Fournal of Thoracic and Cardiovascular Surgery, 65, 364-375.

Zuhdi, N., Hawley, W., Voehl, V., Hancock, W., Carey, J., and Greer, A. (1974). Porcine aortic valves as replacements for human heart valves. Annals of Thoracic Surgery, 17, 479491.

Requests for reprints to Dr J. C. Forfar, Department of Cardiology, The Royal Infirmary of Edinburgh, Edinburgh EH3 9YW. 